\title{
ON LIPSCHITZ HOMOGENEITY OF THE HILBERT CUBE
}

BY

\author{
AARNO HOHTI
}

\begin{abstract}
The main contribution of this paper is to prove the conjecture of [Vä] that the Hilbert cube $Q$ is Lipschitz homogeneous for any metric $d_{s}$, where $s$ is a decreasing sequence of positive real numbers $s_{k}$ converging to zero, $d_{s}(x, y)=\sup \left\{s_{k}\left|x_{k}-y_{k}\right|: k \in N\right\}$, and $R(s)=\sup \left\{s_{k} / s_{k+1}: k \in N\right\}<\infty$. In addition to other results, we shall show that for every Lipschitz homogeneous compact metric space $X$ there is a constant $\lambda<\infty$ such that $X$ is homogeneous with respect to Lipschitz homeomorphisms whose Lipschitz constants do not exceed $\lambda$. Finally, we prove that the hyperspace $2^{I}$ of all nonempty closed subsets of the unit interval is not Lipschitz homogeneous with respect to the Hausdorff metric.
\end{abstract}

1. Introduction. Let $\left(X_{1}, d_{1}\right)$ and $\left(X_{2}, d_{2}\right)$ be metric spaces. A surjective map $f: X_{1} \rightarrow X_{2}$ is called a Lipschitz homeomorphism if there exists a real number $K \geq 1$ such that $K^{-1} d_{1}(x, y) \leq d_{2}(f x, f y) \leq K d_{1}(x, y)$ for all points $x, y$ of $X_{1}$. Let bilip denote the least such constant $K$. In case bilip $f \leq L$, we say that $f$ is an $L$-Lipschitz homeomorphism. A metric space $X$ is called Lipschitz homogeneous if for all points $x$ and $y$ of $X$ there is a Lipschitz homeomorphism $f: X \rightarrow X$ with $f(x)=y$. It is well known that the Hilbert cube $Q=[-1,1]^{N}$ is topologically homogeneous as was shown by $[\mathbf{K e}]$ in 1931. It is natural to ask whether $Q$ is Lipschitz homogeneous with respect to some suitable metric. Let $Q_{s}$ be the Hilbert cube equipped with the metric $d_{s}$, where $s$ is a decreasing sequence of positive real numbers converging to zero and $d_{s}(x, y)=\max \left\{s_{k}\left|x_{k}-y_{k}\right|: k \in N\right\}$. Then $d_{s}$ is a natural metric for the setting of Lipschitz maps. J. Väisälä proved in [Vä] that if $Q_{s}$ is Lipschitz homogeneous, then

$$
R(s)=\sup \left\{s_{k} / s_{k+1}: k \in N\right\}<\infty .
$$

We have proved the converse that was left open.

On the other hand, it was also shown in [Vä] that if one takes $s=\{1 / k: k \in N\}$, then there is a constant $L<\infty$ such that for all $x, y \in Q$ there exists an $L$-Lipschitz homeomorphism $f: Q_{s} \rightarrow Q_{s}$ with $f(x)=y$. However, we shall prove that every compact Lipschitz homogeneous metric space has this property.

2. Notation. Let $S$ denote the set of all decreasing sequences $s$ of positive real numbers $s_{k}$ converging to zero and for which $R(s)<\infty$. We will think of the cube $Q_{s}$ as an infinite product of the line segments $I_{k}^{s}=\left[-s_{k}, s_{k}\right]$ with the natural metric $d_{s}$ given by $d_{s}(x, y)=\left(\|x-y\|_{c_{0}}\right)=\max \left\{\left|x_{k}-y_{k}\right|: k \in N\right\}$. Then $Q_{s}$ can be considered as a metric subspace of the Banach space $c_{0}(N)$. For each $k \in N$,

Received by the editors February 17, 1984.

1980 Mathematics Subject Classification. Primary 54E40.

Key words and phrases. Hilbert cube, homogeneous, Lipschitz homeomorphism, Q-manifold, hyperspace. 
let $Q_{s}^{k}=\prod\left\{I_{j}^{s}: j \geq k\right\}$. Hence, $Q_{s}=I_{1}^{s} \times \cdots \times I_{k}^{s} \times Q_{s}^{k+1}$ for all $k \geq 1$. For a compact metric space $X$ and $\varepsilon>0$, the maximum cardinality of an $\varepsilon$-discrete subset of $X$ will be denoted by $N(X, \varepsilon)$. (A subset $D$ of a metric space $(X, d)$ is called $\varepsilon$-discrete if $d(x, y) \geq \varepsilon$ for all distinct elements $x, y \in D$.) The symbol $\bar{B}(A, \varepsilon)$ denotes the closed $\varepsilon$-neighborhood of a set $A$. The set of all Lipschitz homeomorphisms of $X$ onto itself will be denoted by $L(X)$, and for a real number $r \geq 1$, we write $L_{r}(X)=\{f \in L(X):$ bilip $f \leq r\}$.

3. Lipschitz homogeneity of the cubes $Q_{s}$. In this section we establish the following result, which is the main contribution of the paper.

\subsection{THEOREM. If $s \in S$, then $Q_{s}$ is Lipschitz homogeneous.}

Proof. We shall say that a point $x \in Q_{s}$ is in good position if $x_{k} \geq s_{k}-s_{k+1} / 4$ for each $k \in N$. The meaning of this convention will become clear later. First we shall show that every point of $Q_{s}$ can be mapped to a good position by a Lipschitz homeomorphism of $Q_{s}$. Indeed, let $x \in Q_{s}$. For each $k \in N$, let $f_{k}$ be a PLhomeomorphism $I_{k}^{s} \rightarrow I_{k}^{s}$ such that $\left|f_{k} x_{k}\right| \geq s_{k}-s_{k+1} / 4$. Note that we can assume bilip $f_{k} \leq s_{k} /\left(s_{k+1} / 4\right) \leq 4 R(s)$. If $f_{k} x_{k} \geq 0$, let $g_{k}$ be the identity map of $I_{k}^{s}$. Otherwise let $g_{k}$ be the reflection of $I_{k}^{s}$ relative to the point 0 . Then the map $h: Q_{s} \rightarrow Q_{s}$, given by

$$
h\left(q_{1}, q_{2}, \ldots\right)=\left(g_{1} f_{1} q_{1}, g_{2} f_{2} q_{2}, \ldots\right),
$$

is a $4 R(s)$-Lipschitz homeomorphism of $Q_{s}$ such that $(h x)_{k} \geq s_{k}-s_{k+1} / 4$ for every $k \in N$.

To show that $Q_{s}$ is Lipschitz homogeneous it will be sufficient to prove that an arbitrary point of $Q_{s}$ can be mapped to $\overline{0}=(0,0, \ldots)$ by a Lipschitz homeomorphism of $Q_{s}$. To that end, let $q \in Q_{s}$. By the above, we can assume that $q$ is in good position. For each $k \in N$, we define a map $\varphi_{k}$ of $I_{k}^{s} \times I_{k+1}^{s}$ onto itself as follows. (The square $I_{k}^{s} \times I_{k+1}^{s}$ has the maximum metric.) Let $\phi_{k}$ be a map $\partial\left(I_{k}^{s} \times I_{k+1}^{s}\right) \rightarrow \partial\left(I_{k}^{s} \times I_{k+1}^{s}\right)$ such that $\phi_{k}$ is the identity on

$$
\partial\left(I_{k}^{s} \times I_{k+1}^{s}\right)-\left[\left(\left\{s_{k}\right\} \times\left[0, s_{k+1}\right]\right) \cup\left(\left[s_{k}-s_{k+1}, s_{k}\right] \times\left\{s_{k+1}\right\}\right)\right]
$$

and $\phi_{k}$ maps linearly the segments $\left\{s_{k}\right\} \times\left[0, s_{k+1} / 2\right],\left\{s_{k}\right\} \times\left[s_{k+1} / 2, s_{k+1}\right]$ and $\left[s_{k}-s_{k+1}, s_{k}\right] \times\left\{s_{k+1}\right\}$ onto the segments

$\left\{s_{k}\right\} \times\left[0, s_{k+1}\right], \quad\left[s_{k}-s_{k+1} / 2, s_{k}\right] \times\left\{s_{k+1}\right\} \quad$ and $\quad\left[s_{k}-s_{k+1}, s_{k}-s_{k+1} / 2\right] \times\left\{s_{k+1}\right\}$, respectively. Then $\phi_{k}$ is defined on $\partial\left(I_{k}^{s} \times I_{k+1}^{s}\right)$. Let the restriction of $\varphi_{k}$ to $A_{k}=\left[s_{k}-s_{k+1}, s_{k}\right] \times\left[0, s_{k+1}\right]$ be the radial extension of $\phi_{k}$ relative to the point $\left(s_{k}-s_{k+1}, 0\right)$ and let $\varphi_{k}$ be the identity on the complement of $A_{k}$. The reader will find Figure 1 helpful.

Then $\varphi_{k}$ is a PL-homeomorphism and the action of $\varphi_{k}$ on $B_{2}$ is given by

$$
\varphi_{k}(x, y)=\left(\frac{3}{2} x-y-\left(s_{k}-s_{k+1}\right) / 2, x-\left(s_{k}-s_{k+1}\right)\right) \text {. }
$$

It follows that $\varphi_{k}$ is a Lipschitz homeomorphism; let bilip $\varphi_{k}=L$. (We note that $L$ is a universal constant not depending on the sequence $s$.)

For each $k \in N$, we define a map $g_{k}: Q_{s} \rightarrow Q_{s}$ by setting

$$
g_{k}=\operatorname{id}_{I_{1}^{s} \times \cdots \times I_{k-1}^{s}} \times \varphi_{k} \times \mathrm{id}_{Q_{s}^{k+2} .}
$$


$\left(s_{k}-s_{k+1}, s_{k+1}\right)$

$\left(s_{k}, s_{k+1}\right)$

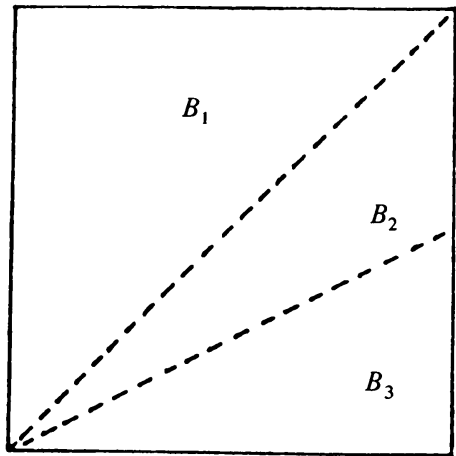

$\left(s_{k}-s_{k+1,0}\right)$

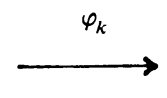

$\left(s_{k}, 0\right)$

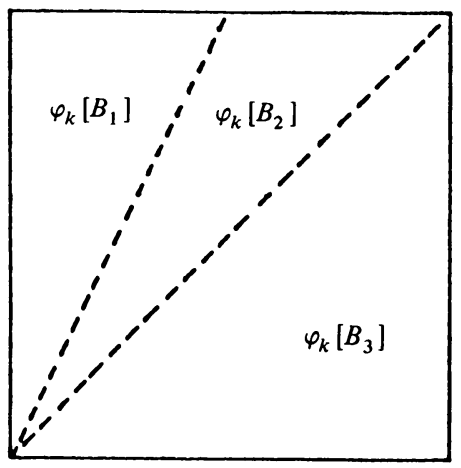

\section{FIGURE 1}

The important property of $g_{k}$ is that it acts "backwards", i.e. the direction of the map is from $I_{k+1}^{s}$-coordinates to $I_{k}^{s}$-coordinates. Further, $g_{k}$ leaves $I_{j}^{s}$-coordinates fixed for $j<k$. Now having defined the maps $g_{k}$, let $f_{n}=g_{n} g_{n-1} \cdots g_{1}$ for $n \in N$. Recall that our point $q$ was in good position in $Q_{s}$. Hence, $\left(q_{1}, q_{2}\right)$ is in good position in $I_{1}^{s} \times I_{2}^{s}$ (in the obvious sense) and thus $f_{1}$ maps $q$ to a point $\left(r_{1}, u, q_{3}, q_{4}, \ldots\right.$ ), where $s_{1}-s_{2} \leq r_{1} \leq s_{1}-s_{2} / 4$ and $s_{2}-s_{3} / 4 \leq u \leq s_{2}$ because $\varphi_{1}$ is level-raising for $I_{2}^{s}$-coordinates. Consequently $\left(\left(f_{1} q\right)_{2},\left(f_{1} q\right)_{3}\right)$ is in good position in $I_{2}^{s} \times I_{3}^{s}$ (see Figure 2). As above we infer that $\left(\left(f_{2} q\right)_{3},\left(f_{2} q\right)_{4}\right)$ is in good position in $I_{3}^{s} \times I_{4}^{s}$ and so on. In general, an inductive argument shows that $f_{n}$ maps $q$ to a point $\left(r_{1}, r_{2}, \ldots, r_{n}, u, q_{n+2}, q_{n+3}, \ldots\right)$, where $s_{i}-s_{i+1} \leq r_{i} \leq s_{i}-s_{i+1} / 4$ for $1 \leq i \leq n$ and $s_{n+1}-s_{n+2} / 4 \leq u \leq s_{n+1}$.

The sequence $\left\{f_{n}: n \in N\right\}$ forms a Cauchy sequence in the space of continuous maps of $Q_{s}$ into itself, equipped with the supremum metric. (Just note that the distance of $f_{n}$ and $f_{n+k}$ is less than $2 s_{n}$ for all $k \in N$.) It follows that $f=\lim f_{n}$ is

$\left(s_{1}-s_{2}, s_{2}\right)$

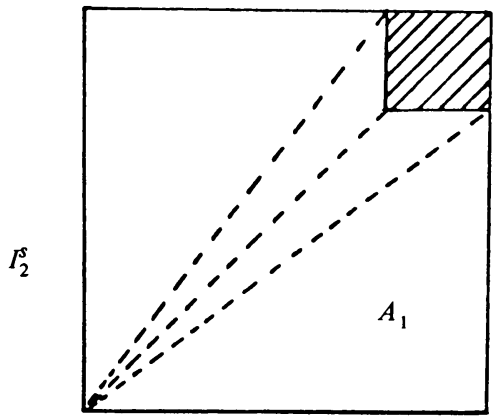

$\left(s_{1}-s_{2}, 0\right)$ $\left(s_{1}, s_{2}\right)$

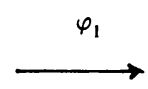

$\left(s_{1}, 0\right)$

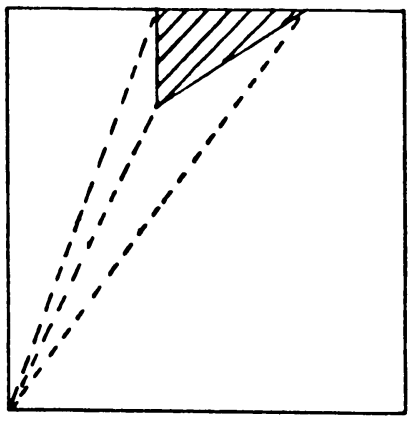

FIGURE 2 
a continuous map of $Q_{s}$ into itself. As $Q_{s}$ is compact, it can easily be shown that $f$ is surjective. We shall prove that $f$ is a Lipschitz homeomorphism.

The proof that $f$ is Lipschitz depends on the following inequality, whose validity is left as an illuminating exercise to the reader. Let $x$ and $y$ be any points of $I_{k}^{s} \times I_{k+1}^{s}$, and let $x^{\prime}=\varphi_{k}(x), y^{\prime}=\varphi_{k}(y)$. Then

$$
\left|x_{k+1}^{\prime}-y_{k+1}^{\prime}\right| \leq \max \left\{\left|x_{k}-y_{k}\right|, 2\left|x_{k+1}-y_{k+1}\right|\right\} .
$$

The idea behind this inequality can be seen from Figure 3 .

Now let $x$ and $y$ be arbitrary points of $Q_{s}$. Put $\|x-y\|_{c_{0}}=\alpha$. We shall prove by induction that $\left|\left(f_{k} x\right)_{k+1}-\left(f_{k} y\right)_{k+1}\right| \leq 2 \alpha$ for all $k \geq 0$, where we put $f_{0}=\mathrm{id}_{Q_{s}}$. Clearly this holds for $k=0$. Suppose that the claim holds for $0 \leq k \leq n$. Since by the induction hypothesis $\left|\left(f_{n} x\right)_{n+1}-\left(f_{n} y\right)_{n+1}\right| \leq 2 \alpha, f_{n}$ does not affect $I_{n+2}^{s}$-coordinates and $\left|x_{n+2}-y_{n+2}\right| \leq \alpha,(*)$ yields

$$
\begin{aligned}
\left|\left(f_{n+1} x\right)_{n+2}-\left(f_{n+1} y\right)_{n+2}\right| & =\left|\left(g_{n+1} f_{n} x\right)_{n+2}-\left(g_{n+1} f_{n} y\right)_{n+2}\right| \\
& \leq \max \left\{\left|\left(f_{n} x\right)_{n+1}-\left(f_{n} y\right)_{n+1}\right|, 2\left|x_{n+2}-y_{n+2}\right|\right\} \leq 2 \alpha
\end{aligned}
$$

Hence, the claim is valid for all $k \in N$. Since the maps $\varphi_{k}$ are $L$-Lipschitz, we obtain $\left|\left(f_{k} x\right)_{i}-\left(f_{k} y\right)_{i}\right| \leq 2 L \alpha$ for all $k \in N$ and $i \leq k$. Therefore, $\left\|f_{k} x-f_{k} y\right\|_{c_{0}} \leq 2 L \alpha$ for every $k \in N$ and thus

$$
\|f x-f y\|_{c_{0}} \leq 2 L\|x-y\|_{c_{0}} .
$$

To prove that $f$ is bilipschitzian, it will be sufficient to show that $f$ satisfies a left-hand side inequality $K^{-1}\|x-y\|_{c_{0}} \leq\|f x-f y\|_{c_{0}}$ for some $K \geq 1$. We shall prove this for $K=2 L$. To accomplish this, we derive from $(*)$ a similar inequality for $\varphi_{k}^{-1}$. This is possible since $\varphi_{k}$ and $\varphi_{k}^{-1}$ have similar expressions on $A_{k}$. (Switch $I_{k}^{s}$ and $I_{k+1}^{s}$, if necessary.) Let $x$ and $y$ be any points of $I_{k}^{s} \times I_{k+1}^{s}$ and let $x^{\prime}=\varphi_{k}(x)$, $y^{\prime}=\varphi_{k}(y)$. Then

$$
\left|x_{k}-y_{k}\right| \leq \max \left\{\left|x_{k+1}^{\prime}-y_{k+1}^{\prime}\right|, 2\left|x_{k}^{\prime}-y_{k}^{\prime}\right|\right\} .
$$

Now let $x, y \in Q_{s}, x \neq y$, and let $k_{0}$ be the least index $k$ for which $\|x-y\|_{c_{0}}=$ $\left|x_{k}-y_{k}\right|=\alpha$. The argument given below shows that we can assume $k_{0}>1$. Since $\varphi_{k_{0}-1}^{-1}$ is $L$-Lipschitz, either

$$
\left|\left(f_{k_{0}-1} x\right)_{k_{0}-1}-\left(f_{k_{0}-1} y\right)_{k_{0}-1}\right| \geq \alpha / L \quad \text { or } \quad\left|\left(f_{k_{0}-1} x\right)_{k_{0}}-\left(f_{k_{0}-1} y\right)_{k_{0}}\right| \geq \alpha / L
$$

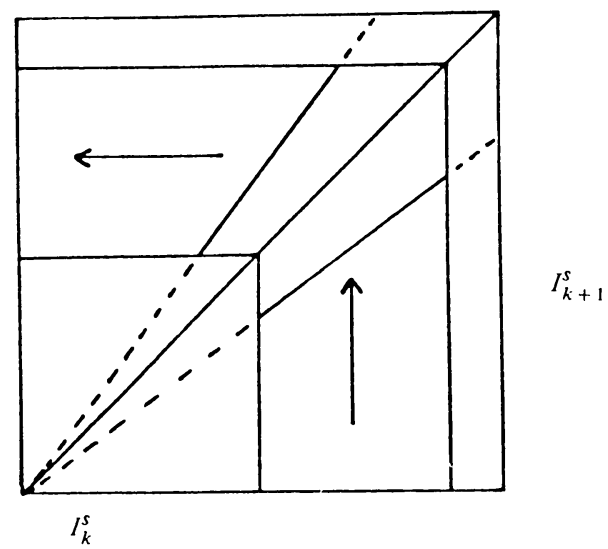

FIGURE 3 
In the first case, $\|f x-f y\|_{c_{0}} \geq \alpha / L$ since $I_{k_{0}-1}^{s}$-coordinates are not changed by $g_{j}$ for $j \geq k_{0}$. Thus, suppose that the latter case occurs. From $(* *)$ we obtain that either

(i) $\left|\left(f_{k_{0}} x\right)_{k_{0}}-\left(f_{k_{0}} y\right)_{k_{0}}\right| \geq \alpha / 2 L$, or

(ii) $\left|\left(f_{k_{0}} x\right)_{k_{0}+1}-\left(f_{k_{0}} y\right)_{k_{0}+1}\right| \geq \alpha / L$.

If (i) is true, then we are done for the same reason as in the first case. Case (ii) is a starting point for an inductive process where (ii) appears at each step. However, case (ii) can occur only finitely many times, since

$$
\left|\left(f_{k} x\right)_{k+1}-\left(f_{k} y\right)_{k+1}\right| \leq 2 s_{k+1} \rightarrow 0 .
$$

It follows that in any case $\|f x-f y\|_{c_{0}} \geq \alpha / 2 L$. Thus, bilip $f \leq 2 L$.

To finish the proof, note that $f$ maps $q$ to the point $\left(r_{1}, r_{2}, \ldots\right)$, where $s_{i}$ $s_{i+1} \leq r_{i} \leq s_{i}-s_{i+1} / 4$. For each $k \in N$, let $h_{k}: I_{k}^{s} \rightarrow I_{k}^{s}$ be a $4 R(s)$-Lipschitz homeomorphism for which $h_{k}\left(r_{k}\right)=0$. Let $h: Q_{s} \rightarrow Q_{s}$ be the map given by

$$
h\left(x_{1}, x_{2}, \ldots\right)=\left(h_{1} x_{1}, h_{2} x_{2}, \ldots\right) .
$$

Then $h f$ is an $8 R(s) L$-Lipschitz homeomorphism of $Q_{s}$ with $h f(q)=\overline{0}$, as required.

REMARK. In particular, $Q$ is Lipschitz homogeneous with respect to the metric $\rho(x, y)=\sup \left\{2^{-k}\left|x_{k}-y_{k}\right|: k \in N\right\}$.

4. The number of essentially different Lipschitz homogeneous metrics $d_{s}$ for $Q$. It is natural to ask what is the number of all Lipschitz equivalence classes of metrics $d_{s}$, where $s \in S$. This question will be answered by using a completely computational application of the following "comparison principle". Recall that for a compact metric space $X$ and an $\varepsilon>0, N(X, \varepsilon)$ denotes the maximum cardinality of an $\varepsilon$-discrete subset of $X$.

COMPARISON PRINCIPLE. Let $X$ and $Y$ be compact metric spaces. If there is an $L$-Lipschitz homeomorphism $\varphi: X \rightarrow Y$, then for each $\varepsilon>0$, we have $N(Y, L \varepsilon) \leq$ $N(X, \varepsilon)$. Thus, if for each $L \geq 1$ there is an $n \in N$ with $N(Y, L / n)>N(X, 1 / n)$, then $Y$ is not Lipschitz homeomorphic to $X$.

For the proof note that if $D$ is an $L \varepsilon$-discrete subset of $Y$, then $\varphi^{-1}[D]$ is an $\varepsilon$-discrete subset of $X$.

4.1. LEMMA. For each $s \in S$ and each $\varepsilon>0$, we have

$$
N\left(Q_{s}, \varepsilon\right)=\prod_{k \in N}\left(\left[2 s_{k} / \varepsilon\right]+1\right)
$$

where $[r]$ denotes the greatest integer $p$ with $p \leq r$.

Proof. For each $k \in N$ let $D_{k}=\left\{-s_{k}+i \varepsilon: 0 \leq i<\left[2 s_{k} / \varepsilon\right]+1\right\}$. Then $D_{k}$ is an $\varepsilon$-discrete subset of $\left[-s_{k}, s_{k}\right]$ with $\left|D_{k}\right|=\left[2 s_{k} / \varepsilon\right]+1$ and hence $D=$ $D_{1} \times D_{2} \times D_{3} \times \cdots$ is an $\varepsilon$-discrete subset of $Q_{s}$ with

$$
|D|=\prod_{k \in N}\left(\left[2 s_{k} / \varepsilon\right]+1\right) .
$$

(Note that the above product makes sense since there is an $n$ such that $2 s_{k} / \varepsilon<1$ for $k \geq n$.) Suppose that $D^{\prime}$ is any $\varepsilon$-discrete subset of $Q_{s}$. For each $k \in N$, we define a map $f_{k}: D^{\prime} \rightarrow D_{k}$ by setting

$$
f_{k}(x)=\max \left\{y \in D_{k}: y \leq x_{k}\right\}
$$


Let $f: D^{\prime} \rightarrow D$ be the map given by $f(x)=\left(f_{1}(x), f_{2}(x), f_{3}(x), \ldots\right)$. Then $f$ is injective. Indeed, suppose that $x, y \in D^{\prime}$ and that $f(x)=f(y)$. For each $k \in N$, we have

$$
x_{k}, y_{k} \in\left[f_{k}(x), f_{k}(x)+\varepsilon[\right.
$$

and consequently $\|x-y\|_{c_{0}}<\varepsilon$ which implies $x=y$ since $D^{\prime}$ is $\varepsilon$-discrete. Thus, $\left|D^{\prime}\right| \leq|D|$.

4.2. Lemma. Let $r, s \in S$ and suppose that $s_{k} / r_{k} \geq 2^{\alpha k}$ for all $k \in N$, where $\alpha>0$. Then $Q_{r}$ is not Lipschitz homeomorphic to $Q_{s}$.

Proof. To derive a contradiction, let $L \geq 1$. Choose an $m^{\prime} \in N$ large enough to satisfy the following conditions: (i) $2^{\alpha m^{\prime} / 4} \geq L$; (ii) $m^{\prime} \geq 4 / \alpha$. Then choose an $n$ such that $1 \leq k \leq m^{\prime}$ implies $r_{k} / 2^{-n-1} \geq 1$ and let $m=\max \left\{k \in N: r_{k} / 2^{-n-1} \geq\right.$ 1). Clearly $m$ satisfies the conditions (i)-(ii) and $2^{n+1} r_{m} \geq 1$. By Lemma 4.1,

$$
N\left(Q_{r}, 2^{-n}\right)=\prod_{k \in N}\left(\left[2^{n+1} r_{k}\right]+1\right)
$$

and

$$
N\left(Q_{s}, 2^{-n} L\right)=\prod_{k \in N}\left(\left[2^{n+1}\left(s_{k} / L\right)\right]+1\right) .
$$

We shall apply the Comparison Principle. For each $k>m$ we have $\left[2^{n+1} r_{k}\right]+1=1$ and thus

$$
\begin{aligned}
\frac{N\left(Q_{s}, 2^{-n} L\right)}{N\left(Q_{r}, 2^{-n}\right)} & \geq \prod_{k=1}^{m} \frac{\left[2^{n+1}\left(s_{k} / L\right)\right]+1}{\left[2^{n+1} r_{k}\right]+1} \geq \prod_{k=1}^{m} \frac{2^{n+1}\left(s_{k} / L\right)}{2^{n+1} r_{k}+1} \geq \prod_{k=1}^{m} \frac{s_{k} / r_{k}}{2 L} \\
& \geq 2^{-m} L^{-m} \prod_{k=1}^{m} 2^{\alpha k}=2^{-m} L^{-m} 2^{m(m+1) \alpha / 2} \\
& \geq\left(2^{-\alpha m / 2} 2^{(m+1) \alpha-2}\right)^{m / 2}=2^{(m \alpha / 2+\alpha-2) m / 2}>1,
\end{aligned}
$$

a contradiction with the assumption that there is an $L$-Lipschitz homeomorphism of $Q_{r}$ onto $Q_{s}$.

4.3. COROLlaRY. There are $2^{\omega}$ essentially different Lipschitz homogeneous metrics $d_{s}$, where $s \in S$.

Proof. For each $\alpha>0$, put $s_{k}(\alpha)=2^{-\alpha k}$. Then $0<\alpha<\beta$ implies $s_{k}(\alpha) / s_{k}(\beta)=2^{(\beta-\alpha) k}$ and Lemma 4.2 shows that $Q_{s(\beta)}$ is not Lipschitz homeomorphic to $Q_{s(\alpha)}$.

5. Lipschitz $Q$-manifolds. As we have shown that the cubes $Q$ are Lipschitz homogeneous for $s \in S$, it is reasonable to introduce the following notion.

5.1. Definition. A separable metric space $X$ is called a Lipschitz $Q$-manifold if there is an $s \in S$ such that each point of $X$ has a neighbourhood which is Lipschitz homeomorphic to $Q_{s}$.

It is well known that a connected Hilbert cube manifold is homogeneous. This can be proved by using the facts that $Q$ is locally homeomorphic to $Q \times[0,1[$ and that every homeomorphism between two $Z$-sets of $Q$ can be extended to a homeomorphism of $Q$. There is no theory of Lipschitz $Z$-sets but we can obtain a 


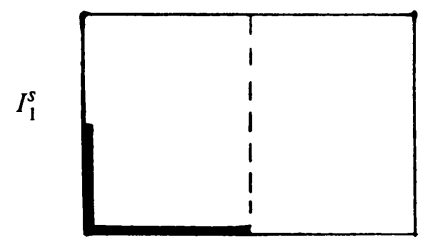

$1 / 4$

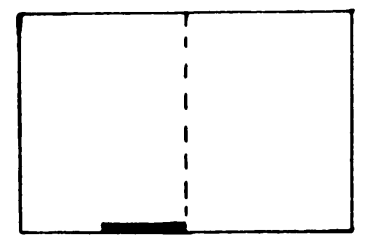

$1 / 4$

\section{FIGURE 4}

proof of the Lipschitz homogeneity of connected Lipschitz $Q$-manifolds by using an elementary isotoping device augmenting the proof of 3.1.

To show that $X$ is Lipschitz homogeneous, it will be sufficient to prove that any point $p \in X$ has a neighbourhood $U$ such that any $q \in U$ can be mapped to $p$ by a Lipschitz homeomorphism of $X$. Since $Q_{s}$ is locally Lipschitz homeomorphic to $\left[0,1\left[\times Q_{s}\right.\right.$ (this follows from 3.1 by an argument similar to that of 12.1 in $[\mathbf{C h}]$ ) it is easily seen that proving the Lipschitz homogeneity of $X$ amounts to establishing the following variant of 3.1 .

5.2. THEOREM. For any two points $x$ and $y$ of $\left[0,1 / 4\left[\times Q_{s}\right.\right.$ there is a Lipschitz homeomorphism $F:\left[0,1\left[\times Q_{s} \rightarrow\left[0,1\left[\times Q_{s}\right.\right.\right.\right.$ such that $F(x)=y$ and $F$ is the identity on $\left[3 / 4,1\left[\times Q_{s}\right.\right.$.

PROOF. We shall show that any point $x$ of $\left[0,1 / 4\left[\times Q_{s}\right.\right.$ can be mapped to the point $(1 / 8, \overline{0})$ by a map $F$ that satisfies the conditions of 5.2 . Since $x_{1}<1 / 4$ there is a Lipschitz homeomorphism of $\left[0,1\left[\times I_{1}^{s}\right.\right.$ which takes $x$ to a point $\left(1 / 8, x_{2}^{\prime}\right)$ and which is the identity on $\left[1 / 4,1\left[\times I_{1}^{s}\right.\right.$. Hence, we can assume that $\left(x_{1}, x_{2}\right) \in\{1 / 8\} \times I_{1}^{s}$. (Figure 4 shows a typical situation when we map a boundary point of $\left[0,1 / 4\left[\times I_{1}^{s}\right.\right.$, i.e. $x_{1}=0$. The map can be realized by taking a suitable PL-homeomorphism of the boundary of $\left[0,1 / 4\left[\times I_{1}^{s}\right.\right.$ and then extending it radially.) From now on we shall work with the projection $x^{\prime}=\left(x_{2}, x_{3}, \ldots\right)$ of $x$ on $Q_{s}$.

As in the proof of 3.1, we use the stretching maps $f_{k}: I_{k}^{s} \rightarrow I_{k}^{s}$ such that $\left|f_{k} x_{k}^{\prime}\right| \geq$ $s_{k}-s_{k+1} / 4$ for all $k \in N$. Since the constants bilip $f_{k}$ are uniformly bounded there is a Lipschitz homeomorphism $F_{1}:\left[0,1\left[\times Q_{s} \rightarrow\left[0,1\left[\times Q_{s}\right.\right.\right.\right.$ which (Lipschitz) isotopes $f_{1} \times f_{2} \times f_{3} \times \cdots$ to the identity so that $F_{1}\left(t, q_{1}, q_{2}, \ldots\right)=\left(t, f_{1} q_{1}, f_{2} q_{2}, \ldots\right)$ for every $(t, q) \in[0,1 / 4] \times Q_{s}$ and $F_{1}$ is the identity on $\left[3 / 4,1\left[\times Q_{s}\right.\right.$. Write $F_{1}(x)=$ $\left(1 / 8, x_{1}^{\prime \prime}, x_{2}^{\prime \prime}, \ldots\right)$.

Following the notation from the proof of 3.1, we must avoid using reflections $g_{k}$ since they cannot be isotoped to the identity. Write $Q_{s}=\left(I_{1}^{s} \times I_{2}^{s}\right) \times\left(I_{3}^{s} \times I_{4}^{s}\right) \times \cdots$. For each $k \in N,\left(x_{2 k-1}^{\prime \prime}, x_{2 k}^{\prime \prime}\right)$ lies in one of the small four corners of $I_{2 k-1}^{s} \times I_{2 k}^{s}$. Let $g_{k}$ be a Lipschitz homeomorphism of $I_{2 k-1}^{s} \times I_{2 k}^{s}$ which maps the corner of $\left(x_{2 k-1}^{\prime \prime}, x_{2 k}^{\prime \prime}\right)$ into the one which is in good position. These maps are to be obtained by rotating the boundary $\partial\left(I_{2 k-1}^{s} \times I_{2 k}^{s}\right)$ (with a suitable squeezing continuing the earlier effect of the maps $f_{k}$ ) and then radially extending to the whole of $I_{2 k-1}^{s} \times I_{2 k}^{s}$. Now one can easily find a Lipschitz homeomorphism $F_{2}:\left[0,1\left[\times Q_{s} \rightarrow\left[0,1\left[\times Q_{s}\right.\right.\right.\right.$ which (Lipschitz) isotopes $g_{1} \times g_{2} \times \cdots$ to the identity so that

$$
F_{2}\left(t, q_{1}, q_{2}, q_{3}, q_{4}, \ldots\right)=\left(t,\left(g_{1}\left(q_{1}, q_{2}\right)\right)_{1},\left(g_{1}\left(q_{1}, q_{2}\right)\right)_{2},\left(g_{2}\left(q_{3}, q_{4}\right)\right)_{1}, \ldots\right)
$$

for all $(t, q) \in[0,1 / 4] \times Q_{s}$ and $F_{2}$ is the identity on $\left[3 / 4,1\left[\times Q_{s}\right.\right.$. 
Next we shall handle the maps $\varphi_{k}$. For each $t \in[1 / 4,3 / 4]$, define $\varphi_{k}(t): I_{k}^{s} \times$ $I_{k+1}^{s} \rightarrow I_{k}^{s} \times I_{k+1}^{s}$ as in the proof of 3.1 but replace $A_{k}$ by the set

$$
A_{k}(t)=\left[s_{k}-2(3 / 4-t) s_{k+1}, s_{k}\right] \times\left[s_{k+1}-2(3 / 4-t) s_{k+1}, s_{k+1}\right] .
$$

Thus, the sets $A_{k}(t)$ are linearly squeezed to their corners. On each slice $\{t\} \times$ $Q_{s}$, where $t \in[1 / 4,3 / 4]$, the maps $\varphi_{k}(t)$ induce a $2 L$-Lipschitz homeomorphism $f_{t}:\{t\} \times Q_{s} \rightarrow\{t\} \times Q_{s}$. Thus, we easily see that we obtain a Lipschitz homeomorphism $F_{3}$ of $\left[0,1\left[\times Q_{s}\right.\right.$ such that the map $f$ from the proof of 3.1 is isotoped by $F_{3}$ to the identity in $[1 / 4,3 / 4] \times Q_{s}$. Let $F_{4}:\left[0,1\left[\times Q_{s} \rightarrow\left[0,1\left[\times Q_{s}\right.\right.\right.\right.$ do the same for the map $h$. Then $F=F_{4} \cdots F_{1}$ is the required map.

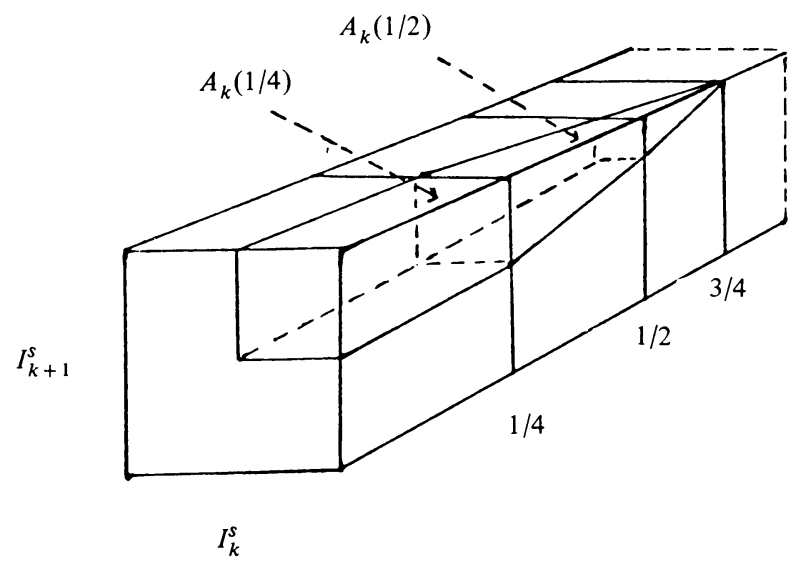

FIGURE 5

5.3. Corollary. A connected Lipschitz Q-manifold is Lipschitz homogeneous.

PROOF. See the proof of the following proposition.

Indeed, Theorem 5.2 yields a slightly stronger "basic cube" result for Lipschitz $Q$-manifolds.

5.4. Proposition. Let $X$ be a connected metric space. Suppose that for each $x \in X$ there exists an $s_{x} \in S$ and a neighbourhood $U_{x}$ of $x$ such that $U_{x}$ is Lipschitz homeomorphic to $Q_{s(x)}$. Then $X$ is a Lipschitz $Q$-manifold.

Proof. As a locally separable connected metrizable space, $X$ is separable. Let $p, q \in X$. Use the connectedness of $X$ to find a simple chain $\left\{U_{1}, \ldots, U_{n}\right\}$ of open subsets of $X$ and points $x_{1}, \ldots, x_{n+1} \in X$ such that $p=x_{1} \in U_{1}, q=x_{n+1} \in U_{n}$ and $x_{i+1} \in U_{i} \cap U_{i+1}$ for $1 \leq i \leq n$, with Lipschitz homeomorphisms

$$
\varphi_{i}: U_{i} \rightarrow\left[0,1\left[\times Q_{s_{i}}, \quad s_{i} \in S,\right.\right.
$$

where $\varphi_{i}\left(x_{i}\right), \varphi_{i}\left(x_{i+1}\right) \in\left[0,1 / 4\left[\times Q_{s(i)}\right.\right.$. By 5.2 we can find a Lipschitz homeomorphism $f_{i}$ of $\left[0,1\left[\times Q_{s(i)}\right.\right.$ such that $f_{i} \varphi_{i}\left(x_{i}\right)=\varphi_{i}\left(x_{i+1}\right)$ and $f_{i}$ is the identity on $\left[3 / 4,1\left[\times Q_{s(i)}\right.\right.$. Define a map $g_{i}: X \rightarrow X$ by setting $g_{i}(x)=\varphi_{i}^{-1} f_{i} \varphi_{i}(x)$ for $x \in U_{i}$ and $g_{i}(x)=x$ for $x \in X-U_{i}$. Let $K_{i}=\varphi_{i}^{-1}\left[[0,3 / 4] \times Q_{s(i)}\right]$, and put $\delta_{i}=d\left(X-U_{i}, K_{i}\right)>0$ and $\mu_{i}=\operatorname{diam} K_{i}$. The map $g_{i}$ being a Lipschitz homeomorphism on $U_{i}$ and the identity on $X-K_{i}$, suppose that $x \in K_{i}$ and $y \in X-U_{i}$. 
Then

$$
1+\frac{\mu_{i}}{\delta_{i}} \geq \frac{d(x, y)+\mu_{i}}{d(x, y)} \geq \frac{d\left(g_{i} x, g_{i} y\right)}{d(x, y)} \geq \frac{d\left(y, K_{i}\right)}{d\left(y, K_{i}\right)+\mu_{i}} \geq \frac{1}{1+\mu_{i} / \delta_{i}},
$$

which shows that $g_{i}$ is a Lipschitz homeomorphism of $X$. Now $g_{n} g_{n-1} \cdots g_{1}$ maps $p$ to $q$. Thus, $X$ is Lipschitz homogeneous and the claim of 5.4 follows.

The use of simple chains in the proof of 5.4 can be motivated by the fact that this proof can easily be modified to show that a connected Lipschitz $Q$-manifold is strongly Lipschitz n-homogeneous for every $n \in N$. It follows that for each $s \in S, Q_{s}$ is strongly Lipschitz $n$-homogeneous. It is well known that if $A_{1}, A_{2}$ are compact subsets of the pseudointerior $\left\{x \in Q_{s}:\left|x_{k}\right|<s_{k} \forall k \in N\right\}$ and $f: A_{1} \rightarrow A_{2}$ is a homeomorphism, then $f$ can be extended to a homeomorphism of $Q_{s}$. The same does not hold for Lipschitz homeomorphisms as can be seen from the following example.

5.5. EXAMPLE. Define a sequence $\left\{\alpha_{n}\right\}$ of positive integers by setting $\alpha_{1}=2$, $\alpha_{n+1}=\alpha_{n}+n^{2}$. Let $a_{n}=s_{n}\left(1-2^{-\alpha_{n}-n}\right)$ and let $b_{n}=s_{n}\left(1-2^{-\alpha_{n}}\right)$. We define two compact subsets $A_{1}, A_{2}$ of the pseudointerior of $Q_{s}$ :

$$
A_{1}=\left\{x \in Q_{s}:\left|x_{k}\right| \leq a_{k} \forall k \in N\right\} \quad \text { and } \quad A_{2}=\left\{x \in Q_{s}:\left|x_{k}\right| \leq b_{k} \forall k \in N\right\} .
$$

Clearly the map $f: A_{1} \rightarrow A_{2}$ given by $(f x)_{k}=\left(b_{k} / a_{k}\right) x_{k}$ is a Lipschitz homeomorphism. Suppose that it has an extension to a Lipschitz homeomorphism $\bar{f}$ of $Q_{s}$. Let $L=\operatorname{bilip} \bar{f}$ and let $n \geq 3$ be such that $2^{n}>L$. Notice that the set $E=\bar{B}\left(A_{1}, 2^{-\alpha_{n}-n} s_{n}\right)$ has the form $F \times Q_{s}^{n}$, where $F$ is the set of all points $x$ of $I_{1}^{s} \times \cdots \times I_{n-1}^{s}$ such that

$$
\left|x_{k}\right| \leq a_{k}+2^{-\alpha_{n}-n} s_{n}<s_{k} \text { for } 1 \leq k<n .
$$

Now $I_{1}^{s} \times \cdots \times I_{n-1}^{s}-F$ is homotopy equivalent to $S^{n-2}$ and so is $Q_{s}-E=$ $\left(I_{1}^{s} \times \cdots \times I_{n-1}^{s}-F\right) \times Q_{s}^{n}$. Since $\bar{f}$ is an $L$-Lipschitz homeomorphism, we have

$$
\bar{B}\left(A_{2}, 2^{-\alpha_{n}-n} s_{n} / L\right) \subset \bar{f}[E] \subset \bar{B}\left(A_{2}, 2^{-\alpha_{n}-n} s_{n} L\right) .
$$

The inequalities $2^{-\alpha_{n}-n} L<2^{-\alpha_{n}}$ and $2^{-\alpha_{n+1}}<2^{-\alpha_{n}-n} / L$ imply that

$$
\bar{B}\left(A_{2}, 2^{-\alpha_{n}-n} s_{n} / L\right)=F^{\prime} \times Q_{s}^{n+1} \text { and } \bar{B}\left(A_{2}, 2^{-\alpha_{n}-n} s_{n} L\right)=F^{\prime \prime} \times Q_{s}^{n+1} \text {, }
$$

where both $F^{\prime}$ and $F^{\prime \prime}$ are products $\left[-r_{1}, r_{1}\right] \times \cdots \times\left[-r_{n}, r_{n}\right]$ with $r_{k}<s_{k}$ for $1 \leq k \leq n$. Thus, there is a natural retraction

$$
\varphi: Q_{s}-\bar{B}\left(A_{2}, 2^{-\alpha_{n}-n} s_{n} / L\right) \rightarrow Q_{s}-B\left(A_{2}, 2^{-\alpha_{n}-n} s_{n} L\right) \quad(L>1 !)
$$

induced by the retraction of $\left(I_{1}^{s} \times \cdots \times I_{n}^{s}\right)-F^{\prime}$ onto $\overline{\left(I_{1}^{s} \times \cdots \times I_{n}^{s}\right)-F^{\prime \prime}}$. Thus, we obtain a retraction

$$
\varphi^{\prime}: Q_{s}-\bar{f}[E] \rightarrow Q_{s}-B\left(A_{2}, 2^{-\alpha_{n}-n} s_{n} L\right) .
$$

The induced map $\varphi_{*}^{\prime}: H_{n-1}\left(Q_{s}-\bar{f}[E], Z\right) \rightarrow H_{n-1}\left(Q_{s}-B\left(A_{2}, 2^{-\alpha_{n}-n} s_{n} L\right), Z\right)=$ $Z$ of the singular groups is an epimorphism and hence $H_{n-1}\left(Q_{s}-\bar{f}[E], Z\right) \neq\{0\}$, which contradicts the fact that $H_{n-1}\left(Q_{s}-E, Z\right)=\{0\}$.

6. The existence of uniform constants of Lipschitz homogeneity. As can be seen from our proofs for 3.1 and 5.4, for each compact connected Lipschitz $Q$-manifold there is a uniform constant $\lambda<\infty$ such that the Lipschitz homogeneity of the manifold is witnessed by Lipschitz homeomorphisms $f$ with bilip $f \leq \lambda$. The following result shows that the same holds for any Lipschitz homogeneous compact metric space. 
6.1. THEOREM. Let $X$ be a Lipschitz homogeneous compact metric space. Then there is a constant $\lambda<\infty$ such that for each pair $x, y$ of points of $X$ there exists an $f \in L_{\lambda}(X)$ with $f(x)=y$.

Proof. For each $x \in X$ and each $n \in N$, let $L_{n}(X ; x)$ denote the set of all $y \in X$ such that there is an $f \in L_{n}(X)$ with $f(x)=y$. First we shall show that the sets $L_{n}(X ; x)$ are closed. Suppose that $y \in X$ and that $\left\{x_{n}\right\}$ is a sequence of elements of $L_{n}(X ; x)$ such that $x_{n} \rightarrow y$. By the definition of $L_{n}(X ; x)$, for each $k \in N$ there is an $f_{k} \in L_{n}(X ; x)$ with $f_{k}(x)=x_{k}$. Since $X$ is compact, $\left\{f_{k}(z): k \in N\right\}$ is relatively compact for all $z \in X$. On the other hand, $\left\{f_{k}\right\}$ is an equicontinuous subset of $C_{c}(X)$ (= the set of all continuous self-maps of $X$ with the topology of compact convergence) since it is even equilipschitzian. By Ascoli's theorem $\left\{f_{k}\right\}$ is relatively compact in $C_{c}(X)$. Hence, there is a subsequence $\left\{f_{k_{j}}\right\}$ of $\left\{f_{k}\right\}$ such that the maps $f_{k_{j}}$ converge uniformly to some $f \in C(X)$. It follows that $f$ is an $n$-Lipschitz homeomorphism of $X$. (Note that $f$ is surjective since $X$ is compact.) But

$$
f(x)=\lim f_{k_{j}}(x)=\lim x_{k_{j}}=y
$$

and hence $y \in L_{n}(X ; x)$. Consequently the sets $L_{n}(X ; x)$ are closed.

Since $X$ is Lipschitz homogeneous, we have $X=\bigcup_{n \in N} L_{n}(X ; x)$ for every $x \in X$. By the Baire Category Theorem there is an $n_{x} \in N$ such that $U_{x}=$ int $L_{n(x)}(X ; x) \neq \varnothing$. Accordingly, choose a point $y_{x} \in U_{x}$. Since $y_{x} \in L_{n(x)}(X ; x)$, there is an $f_{x} \in L_{n(x)}(X)$ with $f_{x}(x)=y_{x}$. As $f_{x}$ is continuous, we can find an open neighbourhood $V_{x}$ of $x$ such that $f_{x}\left[V_{x}\right] \subset U_{x}$. Put $m_{x}=n_{x}^{4}$. It follows that for every pair $z, w$ of points of $V_{x}$ there is an $f \in L_{m(x)}(X)$ with $f(z)=w$. Indeed, let $z, w \in V_{x}$. Then $f_{z}(z), f_{x}(w) \in U_{x}$ and hence (since $U_{x} \subset L_{n(x)}(X ; x)$ ) there exist $g, h \in L_{n(x)}(X)$ such that $g(x)=f_{x}(z)$ and $h(x)=f_{x}(w)$. Consequently the map $f_{x}^{-1} h g^{-1} f_{x}$ takes $z$ to $w$ and

$$
\operatorname{bilip}\left(f_{x}^{-1} h g^{-1} f_{x}\right) \leq n_{x}^{4} .
$$

Now $\left\{V_{x}: x \in X\right\}$ is an open cover of $X$ and has a finite subcover $\left\{V_{x_{1}}, \ldots, V_{x_{n}}\right\}$. For each pair $(i, j)$, where $1 \leq i, j \leq n$, choose a Lipschitz homeomorphism $f_{i j}: X \rightarrow X$ such that $f_{i j}\left(x_{i}\right)=x_{j}$, and let

$$
m=\max \left\{\operatorname{bilip} f_{i j}: 1 \leq i, j \leq n\right\} .
$$

Finally, put $\lambda=m \cdot m_{x_{1}} \cdots m_{x_{n}}$. Then $\lambda$ satisfies the condition of 6.1 . In fact, let $x, y \in X$. Choose $i, j$ so that $x \in V_{i}$ and $y \in V_{j}$. By the previous paragraph there exist $g \in L_{m\left(x_{i}\right)}(X), h \in L_{m\left(x_{j}\right)}(X)$ with $g(x)=x_{i}$ and $h(y)=x_{j}$. Then $h^{-1} f_{i j} g$ maps $x$ to $y$ and

$$
\operatorname{bilip}\left(h^{-1} f_{i j} g\right) \leq m_{x_{j}} \cdot m \cdot m_{x_{i}} \leq \lambda,
$$

as desired.

6.2. REMARK. By an argument that is easier but similar to that given for 6.1, one can show that if $G \subset L(X)$ is a compact group of Lipschitz homeomorphisms of $X$, metrized by the metric $\tilde{\sigma}$ for which

$$
\tilde{\sigma}(f, g)=\max \left\{\sigma(f, g), \sigma\left(f^{-1}, g^{-1}\right)\right\} \quad \text { and } \quad \sigma(f, g)=\sup \{d(f x, g x): x \in X\},
$$

then there is a uniform constant $\lambda<\infty$ such that $G \subset L_{\lambda}(X)$. It was shown in [DW] that there are compact connected 2-manifolds without boundary (any having 
genus $>1$ is good) $X$ such that no compact subgroup of $H(X)$ acts transitively on $X$, or, equivalently, $X$ has no compatible metric for which the isometries are transitive. Anyhow, it has been noted in $[\mathbf{H J}]$ that for any compact homogeneous metric space $X$ and any $\varepsilon>0$, there is a compatible metric of $X$ for which $X$ is Lipschitz homogeneous with a uniform constant $\leq 1+\varepsilon$.

7. The hyperspace $2^{I}$ of closed nonempty subsets of the unit interval. It was shown by Schori and West in 1975 that $2^{I}$ is homeomorphic to $Q$. Their result implied that $2^{I}$ is homogeneous. The following question arises: is $2^{I}$ even Lipschitz homogeneous with respect to its standard Hausdorff metric? It is not too difficult to show that the answer to this question is negative. Recall that the Hausdorff metric (denote it by $d$ ) of $2^{I}$ is defined by setting $d\left(A_{1}, A_{2}\right)<\varepsilon$ iff $A_{1} \subset B\left(A_{2}, \varepsilon\right)$ and $A_{2} \subset B\left(A_{1}, \varepsilon\right)$, where $A_{1}, A_{2} \in 2^{I}$ and $\varepsilon>0$.

7.1. LemMA. For each $n$, we have $N\left(2^{I}, 1 / n\right)=2^{n+1}-1$.

Proof. Let $A=\{i / n: 0 \leq i \leq n\}$. Then $\mathcal{F}=$ the power set $P(A)$ minus the empty set is a $(1 / n)$-net of $2^{I}$. Suppose that $D$ is a $(1 / n)$-discrete subset of $2^{I}$. Define a map $F: D \rightarrow \mathcal{F}$ by setting $x \in F(D)$ iff either $x=0$ and $x \in D$ or $x=i / n$, $i>0$, and

$$
](i-1) / n, i / n] \cap D \neq \varnothing \text {. }
$$

(Then $d(D, F(D))<1 / n$.) Suppose that $D, D^{\prime} \in D$ and $F(D)=F\left(D^{\prime}\right)$. It easily follows that $d\left(D, D^{\prime}\right)<1 / n$ and hence $D=D^{\prime}$. Thus, $F$ is injective which implies $|D| \leq|\mathcal{F}|=2^{n+1}-1$.

\subsection{Proposition. $2^{I}$ is not Lipschitz homogeneous.}

PROOF. We shall prove that there is no Lipschitz homeomorphism of $2^{I}$ mapping the point $\{0\}$ of $2^{I}$ to the point $I$. To derive a contradiction, suppose that $\varphi: 2^{I} \rightarrow$ $2^{I}$ is a Lipschitz homeomorphism with $\varphi(\{0\})=I$ and let bilip $\varphi=L$. Choose an $n \in N$ with $n \geq 5 L^{2}$. Denoting by $\bar{B}_{d}(\{0\}, 1 / n)$ the closed $(1 / n)$-neighbourhood of $\{0\}$ in $2^{I}$, notice that $\bar{B}_{d}(\{0\}, 1 / n)=2^{[0,1 / n]}$ and by the above lemma

$$
N\left(\bar{B}_{d}(\{0\}, 1 / n), 1 / n^{2}\right)=2^{n+1}-1 .
$$

By the Comparison Principle $N\left(\varphi\left[\bar{B}_{d}(\{0\}, 1 / n)\right], L / n^{2}\right) \leq 2^{n+1}$. Since $\varphi$ is $L$ Lipschitz, we have $\bar{B}_{d}(I, 1 / n L) \subset \varphi\left[\bar{B}_{d}(\{0\}, 1 / n)\right]$. Thus, we obtain

$$
N\left(\bar{B}_{d}(I, 1 / n L), L / n^{2}\right) \leq 2^{n+1} .
$$

To see that $(*)$ is not true, let $D=\{i / L n: 0 \leq i \leq r=[L n]\}$, and for $0 \leq i<r$, let $D_{i}$ be an $L / n^{2}$-discrete subset of $] i / L n,(i+1) / L n\left[\right.$ of cardinality $\left[n / L^{2}\right]$. Choose subsets $D_{i}^{\prime} \subset D_{i}$ for which $\left|D_{i}^{\prime}\right| \geq\left[n / L^{2}\right]-2$ and for which $D \cup D_{0}^{\prime} \cup \cdots \cup D_{r-1}^{\prime}$ is an $\left(L / n^{2}\right)$-discrete subset of $I$. (Just throw out the least and greatest elements of $D_{i}$.) Note that any $C \in 2^{I}$ containing $D$ belongs to $\bar{B}_{d}(I, 1 / n L)$. Especially each set $D \cup F_{0} \cup \cdots \cup F_{r-1}$, where $F_{i} \in P\left(D_{i}^{\prime}\right)$, is an element of $\bar{B}_{d}(I, 1 / n L)$. But the collection of all such sets is $\left(L / n^{2}\right)$-discrete in $2^{I}$ and their number is

$$
\prod_{k=0}^{r-1}\left|P\left(D_{k}^{\prime}\right)\right| \geq \prod_{k=0}^{r-1} 2^{n / L^{2}-3}=\left(2^{n / L^{2}-3}\right)^{r}>2^{n+1},
$$

a contradiction. 
Another question which arises in the context of $2^{I}$ is whether $2^{I}$ is Lipschitz homeomorphic to $(Q, \rho)$, where $\rho$ is the metric given by $\rho(x, y)=\sum 2^{-k}\left|x_{k}-y_{k}\right|$. The answer is negative, even if $I$ is replaced by any Peano continuum. (It was shown in $[\mathrm{CS}]$ that if $X$ is a nondegenerate Peano continuum, then $2^{X}$ is homeomorphic to $Q$.)

7.3. Lemma. Let $X$ be a connected metric space with $\operatorname{diam} X \geq 1$. Then $N(X, 1 / 2 n) \geq n$.

Proof. Let $x_{0} \in X$ be arbitrary. Since $X$ is connected and diam $X \geq 1$, for each $j \leq n-1$ there is an $x_{j}$ such that $d\left(x_{0}, x_{j}\right)=j / 2 n$. The set $\left\{x_{0}, \ldots, x_{n-1}\right\}$ is $(1 / 2 n)$ discrete, because $1 \leq i<j \leq n-1$ implies $d\left(x_{i}, x_{j}\right) \geq d\left(x_{j}, x_{0}\right)-d\left(x_{i}, x_{0}\right) \geq 1 / 2 n$.

Now let $X$ be any Peano continuum. We can assume that $\operatorname{diam} X \geq 1$. Let $s$ be the sequence $\left\langle 2^{-k}\right\rangle$. Note that if $x, y \in Q$ and $\rho(x, y) \geq 2^{-n}$, then there is an $i \leq n+1$ with $\left|x_{i}-y_{i}\right| \geq 2^{-n-1}$. Therefore, $s_{i}\left|x_{i}-y_{i}\right| \geq 2^{-2 n-2}$ and hence $d_{s}(x, y) \geq 2^{-2 n-2}$. It follows that any $2^{-n}$-discrete subset of $(Q, \rho)$ is $2^{-2 n-2}$ discrete in $Q_{s}$ and so

$$
N\left((Q, \rho), 2^{-n}\right) \leq N\left(Q_{s}, 2^{-2 n-2}\right) \leq 2^{(n+3)(2 n+3)},
$$

where we have used Lemma 4.1. However, $N\left(2^{X}, 2^{-n}\right)$ grows much faster. Indeed, by Lemma $7.3, N\left(X, 2^{-n}\right) \geq 2^{n-1}$ and thus $N\left(2^{X}, 2^{-n}\right) \geq 2^{2^{n-1}}-1$. Consequently,

$$
N\left(2^{X}, 2^{-n} L\right) / N\left((Q, \rho), 2^{-n}\right) \rightarrow \infty \quad \text { as } n \rightarrow \infty
$$

for any $L \geq 1$ and the Comparison Principle shows that $2^{X}$ is not Lipschitz homeomorphic to $(Q, \rho)$.

ACKNOWLEDGEMENT. The author is grateful to J. Liukkonen, J. Väisälä and J. Luukkainen for several useful comments.

\section{REFERENCES}

[Ch] T. A. Chapman, Lectures on Hilbert cube manifolds, CBMS Regional Conf. Ser. in Math., No. 28, Amer. Math. Soc., Providence, R.I., 1976.

[CS] D. W. Curtis and R. M. Schori, Hyperspaces of Peano continua are Hilbert cubes, Fund. Math. 101 (1978), 19-38.

[DW] D. van Dantzig and B. L. van der Waerden, Ueber metrisch homogene Räume, Abh. Math. Sem. Univ. Hamburg 6 (1928), 367-376.

[HJ] A. Hohti and H. Junnila, A note on homogeneous metrizable spaces, manuscript.

[Ke] O. H. Keller, Die Homoiomorphie der kompakten konvexen Mengen in Hilbertschen Raum, Math. Ann. 105 (1931), 748-758.

[SW] R. M. Schori and J. E. West, The hyperspace of the closed unit interval is a Hilbert cube, Trans. Amer. Math. Soc. 213 (1975), 217-235.

[Vä] J. Väisälä, Lipschitz homeomorphisms of the Hilbert cube, Topology Appl. 11 (1980), 103-110.

Department of Mathematics, University of Helsinki, Hallituskatu 15 , SF00100 HELSINKI 10, FINLAND 